\title{
NSNS RING BEAM DIAGNOSTIC INSTRUMENTATION
}

\section{BNL/NSNS TECHNICAL NOTE}

\author{
NO. 017
}

\author{
R. Witkover
}

February 14, 1997

ALTERNATING GRADIENT SYNCHROTRON DEPARTMENT BROOKHAVEN NATIONAL LABORATORY UPTON, NEW YORK 11973 


\section{NSNS Tech Note 17 \\ NSNS Ring Beam Diagnostic Instrumentation}

R. Witkover

February 1997

\section{INTRODUCTION}

The National Spallation Neutron Source (NSNS) is designed to provide a short pulse of 1 $\mathrm{GeV}$ protons at an intensity of $1-2 \times 10^{14}$. At the design repetition rate of $60 \mathrm{~Hz}$ this will result in an average beam power of 1-2 MW. The accelerator systems will consist of a $1 \mathrm{GeV}$ linac feeding an accumulator ring which will convert the 1 millisecond long linac beam pulse, injected over 1200 turns, into the 500 nano-second output pulse. The high average beam power presents significant problems in the design of the detectors and makes the need for significant beam instrumentation to minimize beam loss imperative. From both beam loss and instrumentation survival considerations, non-intercepting monitors are necessary. The designs described here have been upgraded to the fourfold symmetry lattice. Figure 1 shows the layout of instrumentation in the ring, which is also summarized in Table 1. This note will describe some of the instrumentation and the considerations that went into the selection. For more information about other NSNS systems and design, consult the NSNS Technical Notes.

\section{BEAM LOSS MONITORS}

Because of the high average beam power, only a very small percentage loss will be acceptable in the NSNS Ring, otherwise machine components would become activated and materials would deteriorate requiring more frequent maintenance. High background levels would make maintenance difficult and hazardous. Careful monitoring of beam loss will be necessary to keep the radiation dose at tolerable levels. A loss of 1 part in $10^{4}$, representing between 1 and $2 \times 10^{10}$ protons at the design intensity, would be considered tolerable. While it is possible to measure significant beam losses using a Beam Current Monitor (BCM), the small differences of interest in a high average power machine would be hard to resolve in practice, particularly if occurring slowly over time. A much better approach is to measure the radiation produced by the particles lost, since this is a deviation from zero and more discernable. Ion chambers, scintillator-photo-multipliers or solid-state radiation detectors have been used for loss measurement. The pros and cons of these different types of detectors will be discussed below. Among the considerations are: cost, stability, unit-to-unit reproducibility, sensitivity, dynamic range, speed of response, resistance to radiation damage and low maintenance. Neutron detection is not normally part of the accelerator diagnostics system but may be included as part of the safety system.

The primary functions of the Beam Loss Monitor system are to minimize losses by allowing tuning of the beam and preventing injection of subsequent beam following a high loss. Tuning requires that the loss data be continuously acquired and displayed. Programmable threshold detection circuitry will provide an interlock signal to prevent beam injection following high loss pulses. This 
loss monitor system will not be used for personnel protection, which requires fail-safe design and high level certification.

A number of different detectors have been used for beam loss monitoring at various accelerators. These include: ion chambers, scintillator-photo-multipliers and solid-state radiation detectors. Each has advantages and disadvantages, which will be discussed here.

\section{Scintillator-Photomultipliers}

At FNAL, "Paint Cans" were used as the radiation monitors for some years. The PSR also uses this type detector to track fast losses. These got their names from the container for the liquid scintillator chosen to reduce the effects of radiation darkening. Scintillator-Photo-multipliers offer a wide dynamic range and fast (nano-second) response. Significant unit-to-unit variation and radiation darkening of the scintillator and the glass of the photo-multiplier tube requires that the calibration be periodically checked and individual controllable HV power supplies be used. The cost of the tubes and their bases is significant.

\section{Solid State Radiation Detectors}

Solid state junctions produce a signal when exposed to ionizing radiation. There are compact and simple to employ. Typical sensitivity is $20 \mathrm{nC} / \mathrm{Rad}$. A novel implementation at DESY ${ }^{1}$ uses dual detectors in a mini telescope to discriminate against synchrotron radiation background from their electron ring. They are simple to use, require no bias supply and are very compact. However. They have low sensitivity and are highly vulnerable to radiation damage (better suited for low radiation environment)

Ion Chambers: Ion chambers consist of a gas filled enclosure with a pair or pairs of positive and negative electrodes. Ionizing radiation generates ion-electron pairs along its path, producing a signal as the charges move in the electric field. Argon is used in most accelerator beam loss monitoring applications because it is inert, inexpensive even in high purity, and free of electronegative carriers such as oxygen which would drastically reduce electron mobility. In some cases small percentages of other gasses such as methane are added to enhance the response time. As the electric field is increased more ion-electron pairs are collected until the plateau region is reached, where all created are collected. The voltage range of the plateau depends on the geometry, gas and pressure. With increasing incident radiation the voltage necessary for complete collection increases, extending the knee region as the plateau width shrinks.

The most common Ion Chamber designs are built with a cylindrical coaxial geometry, which offers simple construction, or as parallel plates, which can provide faster response time, particularly for larger volumes. Ion chambers can be made to operate in the proportional mode in which they exhibit "gain" when the electric field is large enough that the energy gained between collisions allows further ionization. Gains of $10^{3}$ are practical, however, the NSNS Ring should not require gain to observe $10^{-4}$ losses.

A beam loss of 1 part in $10^{4}$ would represent between 1 and $2 \times 10^{10}$ protons at the design intensity. In the AGS Booster losses smaller than this have been observed at energies between 600 
$\mathrm{MeV}$ and $1.3 \mathrm{GeV}$. Long coaxial chambers, made from air-dielectric coax cable, have been used at the AGS in the Linac ${ }^{2}$, Booster $^{3}$, and AGS ring ${ }^{4}$. These have the advantage of continuous end-to-end coverage and $1 / r$ response, but require a supporting gas system with its inherent maintenance. Sealed glass units have been developed for the Tevatron at FNAL ${ }^{5}$ and are also used in the transfer line to RHIC and in the RHIC Ring. The sensitivity of these units is $70 \mathrm{nC} / \mathrm{Rad}$. While they are "point"detectors with a $1 / r^{2}$ fall off with distance from the loss, effective coverage can be provided by distributing them at all likely loss points such as quadrupole magnets (points of maximum beta), injection and extraction regions and at scrapers. Although the long beam loss monitors have a larger volume and therefore higher sensitivity, the small FNAL units can be located closer to the beam pipe where the higher radiation results in similar signal levels. The Tevatron type detectors operate at a nominal $1500 \mathrm{~V}(3000 \mathrm{~V}$ max) compared to $200 \mathrm{~V}$ on the coax cable type detectors. This significantly raises the radiation level at which incomplete collection occurs (which has been a concern with the coax cable units). Response time ranges from 1-2 microseconds for electrons to 1-2 milliseconds for ions.

The Tevatron style Ion Chambers offer reasonable response time, low cost ( $\$ 200$ assembled), unit-to-unit reproducibility, long term stability and low maintenance. Sensitivity is also in the right range. Non-linearity can occur for very high loss but levels of interest here are well below this.

Based on the advantages and disadvantages of the above detectors Ion Chambers are the clear choice for the Beam Loss Monitors in the NSNS Ring. Since most accelerators today have made a similar choice, there seems to be general acceptance except where local peculiarities preclude it. The FNAL type sealed glass chambers will be used, with the modifications developed for the RHIC design. These include the use of non-Teflon connectors and isolated BNCs to break signal ground loops.

\section{Location of Detectors}

One Beam Loss Monitor (BLM) will be provided for each quadrupole in the ring, since the beam is largest (beta-max) at those points. Additional units will be located at injection and extraction. A number of relocatable units will be provided which will be placed as operational requirements dictate for a total of 80 channels. The detectors will be mounted as close to the beam pipe as convenient, possibly directly on the vacuum flanges as in the RHIC transfer line.

A number of scintillator-photomultipliers (SPMs) will also be installed. One unit will be located at the downstream end of each straight section. Several SPMs be located in areas where timeresolved knowledge of fast losses might provide information, such as at injection and extraction, where clipping of the bunch tails due to mis-timing or losses due to beam in the gap must be observed. These will also be useful for observing losses due to instabilities should they occur. The units would be integrated over the duration of the bunch and read through VME based fast digitizer channels clocked at the revolution frequency. In addition, all of the units at a given location would be buffered and selectable through a wideband mux to be read into a VME fast digitizer of 100 Megasamples/second or more to observe losses within the bunch duration. 


\section{Electronics}

Various types of signal conditioning electronics have been used. In cases where fast loss detection is required only the electron signal is processed, cutting the sensitivity in half, but allowing 1-2 $\mu \mathrm{sec}$ response. In the BNL Linac the electron signal is amplified and sampled and held for computer acquisition and display, while a comparator, which is active during the full pulse senses excessive loss. This provides continuous loss protection but only discrete time sampling of the losses. The AGS Booster and Ring use integrators which are reset periodically through the acceleration cycle, effectively extending the dynamic range of the electronics. Sample-and-Holds buffer the signals until the control system can acquire the data. At the Tevatron logarithmic integrators are used since the wide signal range must be mapped into an 8-bit ADC. The RHIC transfer line uses integrators to capture the losses from the single bunch transfer. Both electrons and ions are collected. In the electronics designed for the RHIC Rings, amplifiers with response-times tailored to the superconducting magnet's thermal time constants acquire the signal which is then digitized by a 14-bit ADC at a $720 \mathrm{~Hz}$ rate. Threshold detectors monitor the loss information and provide a trip signal within $10 \mu \mathrm{s}$ when a programmable level is exceeded, allowing the beam to be dumped or inhibited if desired.

To achieve the desired signal response only electrons will be collected. The electronics will consist of a fast integrator preceded by a buffer amplifier which will match the cable and provide gain adjustment. The integrators would be followed by commercial eight-channel VME ADCs, eliminating the need for Sample-and-Holds. These modules also provide on board FIFO memories sufficiently deep for many seconds data storage to allow playback following a beam fault. At the end of each pulse the front end computer (FEC) will sum the data for each channel (120 readings) to get a single value for the loss on that cycle. Comparators with programable reference levels will monitor the losses on each channel and may be used to inhibit further injection. A system similar to this has been in use in the AGS Linac for many years.

\section{BEAM CURRENT MONITORS}

The circulating current in the ring will be monitored using a Beam Current Transformer (BCM). In the simple current transformer the beam, passing through the aperture of a high permeability tape wound core, acts as a single turn primary winding with the signal developed on a secondary winding. Since the output depends on the time derivative of the current, care must be taken to properly integrate the signal to reconstruct the beam current. In applications where the transformer inductance " $L$ " and the load resistance " $R$ " are such that $L / R>>$ the beam pulse width and the duty factor is low, the signal can be passively integrated. If DC response is required, as in a storage ring, a transductor (true DC transformer or DCCT) can be used. In other cases where the simple transformer's low frequency response is inadequate but the complexity of a DCCT is not justified, magnetic feedback can be used. As for the simple transformer, the beam forms the primary winding and the signal is picked up by the secondary winding and amplified. In this case it is coupled back into the transformer through a tertiary winding to compensate the droop. Using this technique, developed by Hereward ${ }^{6}$, droop time constants well over 100 seconds may be achieved. 


\section{BCM Parameters}

In the NSNS Ring the beam consists of $1-2 \times 10^{14}$ charges injected into a single bucket over 1200 turns. Since the peak current in the bunch at the end of injection will be from 20 to over 100 A (depending on bunch shape) care must be taken in the transformer design to prevent saturation of the core. To observe the beam during the approximately 1 millisecond long accumulation period with less than $0.1 \%$ droop would require a decay time constant of more than 1 second, which is quite practical with a magnetic feedback transformer. However, if the droop compensation feedback is made too strong the upper cut-off frequency may be too low to allow the turns to be cleanly separated. A rise time of less than $50 \mathrm{nsec}$ would be needed to see the bunch with reasonable fidelity. This might be difficult to obtain with the competing requirement for low droop. However, high frequency beam current information can be obtained from the Wall Current Monitor or a separate high frequency BCM. It is possible to extend the bandwidth on a single transformer by combining the slower magnetic feedback circuitry with the faster simple transformer to merge the best characteristics of each. ${ }^{7}$ On the other hand it may be desirable to keep the bandwidth of the BCM limited so that the gap in the beam current does not confuse the data acquisition. At the PSR the $\mathrm{BCM}$ rise time is about $10 \mu \mathrm{sec}^{8}$.

A magnetic feedback transformer as described above would be the best choice for the NSNS Ring. A rise time of $5 \mu \mathrm{sec}$ and a decay time constant of 10 seconds would be sufficient. Several decades of gain switching will be provided to allow measurement of short Linac pulses for studies as well as full beam. Data acquisition would be as an additional channel in the same ADC modules as the BLM data, taken every 10 microseconds or at specific times within the cycle.

The $\mathrm{BCM}$ will be located in a straight section in a 0.3 meter section of beam pipe. A ceramic break must be included to eliminate image currents.

\section{Fast Current Monitor}

The bunch longitudinal density distribution must be monitored to provide information for the $\mathrm{RF}$ system, for detection of high frequency instabilities, and for the calculation of momentum spread. The choices are between a fast Bram Current Transformer (BCM) and a Wall Current Monitor (WCM). The WCM makes use of the image current which flows along the beam pipe and is exactly equal in amplitude and opposite to the beam current, can have a frequency response from $6 \mathrm{GHz}$ to $3 \mathrm{kHz}^{9}$. The upper frequency response of this unit was achieved by designing the gap as a radial transmission line. The low frequency cut-off is limited by the inductance in the loop formed by the outer shield, and will set the rate of baseline droop. The $3 \mathrm{KHz}$ low frequency roll off will cause the baseline of the signal to drift significantly during the $1 \mathrm{msec}$ pulse, coming to rest such that the average value of the signal bunch signal is near zero. This may be a serious limitation since the low frequency response cannot be significantly extended.

The other alternative would be a Fast Beam Current Transformer (FBCT). There is a commercially available unit which has a 600 picosecond risetime and less than $1 \%$ droop per microsecond. The unit can operate at up to 2000 A pulses provided the RMS current is less than 28 A. 
Because of the poor low frequency response of the WCM, the FBCT will be used to obsert the longitudinal density distribution. The data will be acquired using a fast VME transient digitizer of 100 Mega-samples/second or greater, with sufficient local storage to record a history over many seconds. A second unit will be installed for RF timing purposes.

The Fast Current Monitor will be located in a straight section in a 0.5 meter section of beam pipe.

\section{Beam In Gap (BIG) Monitor}

It will be desirable to monitor the beam outside the bucket to minimize losses at extraction and help in setting the RF. There may be only a small fraction of the beam outside the bucket but even $0.1 \%$ lost around the machine would be unacceptable. This means that we must be able to resolve 4-5 orders of magnitude below the peak in the bunch without saturating the detector or electronics. A clever scheme is used at the PSR in which the extraction kicker is timed to fire in the gap so that only that beam goes out of the machine ${ }^{10}$. Then the standard instrumentation is able to see the small signal without being swamped by the main beam. A disadvantage of the method is that the full beam is lost in the machine, so it cannot be used as an on-line measurement.

Several other methods should be considered. A separate beam current transformer which can be rapidly gain switched to prevent saturation of the electronics during the bunch might be feasible. Care must be taken to prevent the core from saturating due to the main bunch current. A fast transformer will not have the low frequency response to prevent a baseline shift due to droop during the 1 millisecond cycle. Without a well defined true zero it would be hard to tell if the baseline offset was due to the AC coupling or tails of the beam in the bucket. It might be possible to put the fast transformer windings on the same core as a magnetic feedback transformer but not combine the two signals in the electronics. In this way only the fast transformer could be gain switched at the revolution frequency while the slower transformer would keep the flux in the core from decaying.

A second approach might employ a second extraction kicker which would be timed to fire a short pulse timed for the gap just prior to extraction of the full bunch. The kicked beam could be sent to a separate diagnostic extraction port or down the normal extraction channel where a WCM or fast $\mathrm{BCM}$ could observe the beam with high sensitivity. The kicker for the BIG monitor would require a rise time of 20-30 nsec to prevent encroaching on the tails of the bunch beam. A traveling wave magnet design would probably be required. If the main bunch kicker is designed to be fast enough and the problem of having two separate pulsers connected to the same magnet can be solved then the cost would be minimized. Otherwise this approach would be as expensive as the extraction system and probably not practical.

Another possibility might be to build a BIG detector using residual gas ionization. As the beam passes through the vacuum it ionizes molecules along its path in proportion to the beam density. If a sufficiently large electric field can be applied to overcome the beam space charge field then these ion-electron pairs will be swept to the electrodes. The Ionization Profile Monitor ${ }^{11}$ makes use of this technique to observe the beam transverse density distribution. Since the electron transit times can be on the order of 1-2 nsec, information about the longitudinal density can also be obtained. To reduce the applied electric field requirements a parallel magnetic field is used to trap the electrons on the field 
lines and prevent spreading of the distribution. In the present case since transverse information is not of interest, only a single collector is needed rather than the normal multichannel array, and, a stabilizing magnetic field is probably not required. The output signal could then be processed by a commercial fast logarithmic amplifier such as the AD640 with $100 \mathrm{Mhz}$ bandwidth, to compress the dynamic range between the 10's of Amps in the bunch and the 10's of milliAmps in the gap.

the Beam In Gap monitor.

A section of vacuum pipe 0.3 meters long has been reserved in a straight section for

Further study of each of these will be required.

\section{Closed Orbit Measurements}

The closed orbit system is one of the primary studies and set-up tools. There will be a dual plane detector at each of the 48 quadrupoles. Two kinds of BPMs are being considered: split plate (capacitive) pickups and stripline detectors. Capacitive pickups sense the charge induced on each of the split plates, which is proportional to the beam location. Following an analog front end amplifier, the signal will be integrated and digitized. A simple difference over sum computation yields the beam position. These devices are linear over a wide dynamic range. The disadvantages include sensitivity to beam loss and higher impedance presented to the beam. Since the low frequency response will be affected by the read-out impedance either a transformer or vacuum tube buffer must be used to match the detector to the cable impedance. Capacitive pickups may also be used as electron clearing electrodes, but this makes the impedance matching problem more difficult and expensive since the transformer or buffer electronics must hold off this voltage. Stripline detectors offer a low beam impedance and less sensitivity to beam loss and are easily matched to the read out cable. Experience with the RHIC design will be used as much as possible.

The analog electronics will consist of an impedance matching front end followed by a gated integrator driven at the revolution frequency. Gain switching will be provided, which together with the typical signal to noise of the detector, will provide sufficient dynamic range for beams from a single injected bunch to 1200 injected turns. Triggering should be trivial since the RF is of constant frequency. Delay modules with separate channels for each BPM and several nano-second settability will trigger the integrators and ADCs. A commercial $4 \mathrm{MHz}$ ADC board with 8 independently acquired inputs and sufficient FIFO memory to store several seconds history will be used to acquire the data. Upon request averaged, single revolution or the full memory data (useful following a beam inhibit/dump may be obtained. Actual position data will be computed in the high level programming.

The BPMs will be located between the dipole and quadrupole, nestled under the coil ends. It will be keyed to the quadrupole for positive location.

Further study will be required. The decision may depend upon the need for clearing electrodes if space does not permit separate units to be installed. Design details will be presented in a separate tech note. 


\section{Fractional Tune Measurement}

Small angle beam kickers will be installed for both planes to ping the beam for measurement of the fractional tune. Readout of the full-cycle memory from the BPMs one-quarter wavelength downstream of the kickers will provide the data for an FFT analysis to yield the tune. Later if a Damper is required the tune measurement will be incorporated into its design as in the AGS. A section of beam pipe in a straight section 2 meters long has been reserved for a tune meter/damper.

\section{Beam Profile Monitor}

Knowledge of the profile and the beta-function at that location allows the emittance to be calculated. Injection errors due to poor painting or mismatch, and instabilities which will cause emittance growth through the cycle can also be observed. Interceptive techniques using scrapers or thin carbon wires rapidly moved across the beam pipe (Flying Wires) may cause too much loss and soon be destroyed by the beam, but will be fully studied before a final design is selected.

An ionization profile monitor make use of the interaction of the beam with residual gas molecules in the vacuum chamber. Depending on the bias voltage polarity, the ions or electrons generated are accelerated to an array of collectors which provide the profile information. To prevent the high space charge field due to the beam from spreading the profile an electric field much stronger than that from space charge must be applied. With large apertures, such as in the NSNS Ring, this may lead to high voltages. If electrons are collected then a parallel magnetic field may be used to keep the electrons confined within the Larmor radius. This is the approach which is being taken in RHIC where the space charge problem will be even more severe.

For an applied field of $2500 \mathrm{~V} / \mathrm{cm}$, ions will have a typical transit time of $0.48 \mu \mathrm{sec}$ while electrons will be collected in $2.1 \mathrm{nsec}$, so electron collection will be required for turn-by-turn measurements While in the beam the electrons will be displaced by the field and acquire a transverse velocity. For a gaussian density distribution, using the values of $2 \times 10^{14}$ charges, a beta max of 16 and an emittance of $120 \mathrm{~mm}$-mrad, the electric field will reach a maximum of $990 \mathrm{~V} / \mathrm{cm} .{ }^{12}$ For an applied field of $2500 \mathrm{~V} / \mathrm{cm}$ the electrons will have a transverse velocity of $1 \times 10^{9} \mathrm{~cm} / \mathrm{sec}$ as a result of the bunch charge distribution. This will result in significant profile broadening unless a stabilizing magnetic filed is appiied parallel to the magnetic field. In this case the transverse velocity of the electron will result in cyclical motion around the magnetic field lines at the Larmor radius. The transverse velocity can come from either the energy acquired by the electron at birth or the ExB drift. Most electrons have a fairly low initial energy at ionization, but a small number suffer hard collisions and are quite energetic. Because they are so few their contibution will be ignored. If we assume a mximum energy at ionization of $10 \mathrm{eV}$ then the electrons will be traveling at $1.88 \times 10^{6} \mathrm{M} / \mathrm{sec}$, for a Larmor radius of $0.1 \mathrm{~mm}$. The ExB drift velocity will be about half this value for a Larmor radius of $0.05 \mathrm{~mm}$. To cover a range of $160 \mathrm{~mm}$ with 64 channels, each channel will be about $2.5 \mathrm{~mm}$ wide, much larger than the Larmor radius. Thus a 0.1 Tesla magnetic field and a $50 \mathrm{kV}$ potential over 20 $\mathrm{cm}$ will be sufficient.

For a vacuum of $1 \times 10^{-9} \mathrm{Torr}$, the center electrode will see a signal of 4.4 nano-Amp for 10 $\mathrm{cm}$ long collectors. This will be too small for reliable measurements in a single turn. Microchannel plates can provide a gain of $10^{5}$ making the signal sufficient for single turn measurements. Provisions 
to gate the microchannel plate bias voltage will be required to prevent plate burn out. Care must also be taken to surpress secondary emission due to ions striking the high voltage bias plate. These will be accelerated towards the MCP and arrive with a very divergent profile, giving a false halo to the collected beam signal. This can be avoided by placing a mesh or grid in front of the HV electrode biased some $10 \mathrm{~V}$ or so negative with respect to the HV potential.

The use of a stabilizing magnetic field will cause a deflection of the circlating beam unless compensating magnets are located on either side. Since the field is about $10 \%$ of the typical dipole field it is not likely that the normal correction dipoles could provide this and a separate set must be installed. If the horizontal and vertical IPM planes are located in the same straight section then a single pair of compensating magnets at 45 degrees might be sufficient.

An alternative might be to measure the pickup on a set of electrodes distributed in angle around the beam pipe ${ }^{13}$. The field detected might be able to provide sufficient information about the moments of the transverse density distribution.

A 1 meter section of beam pipe in a each of 2 straight sections has been reserved for the profile monitors.

Further study will be required. 
Figure 1

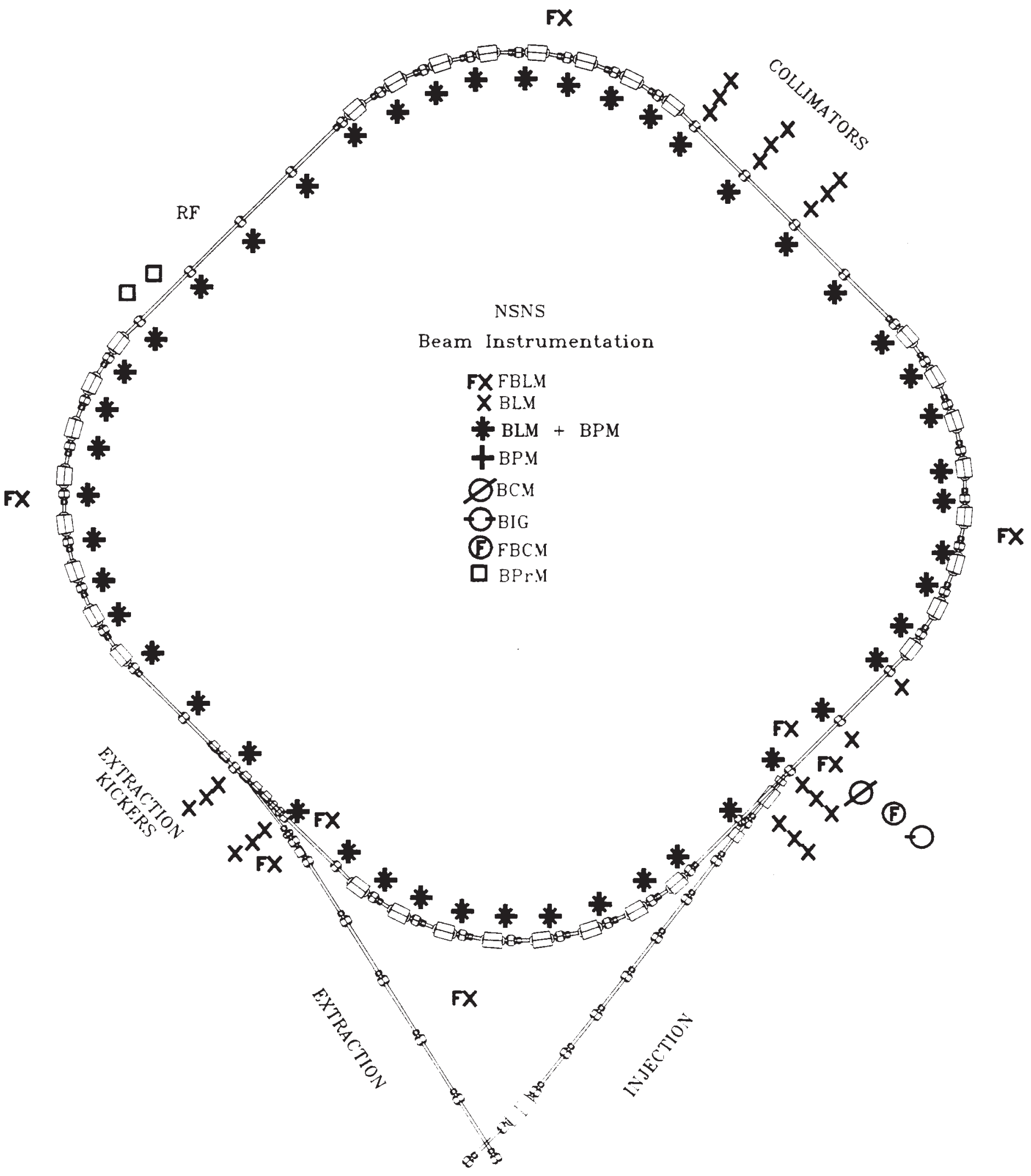




\section{RING BEAM INSTRUMENTATION}

\begin{tabular}{|c|c|l||}
\hline DEVICE & QUANTITY & \multicolumn{1}{|c|}{ DESCRIPTION } \\
\hline Beam Position Monitors & 96 planes & $\begin{array}{l}\text { Dual plane beam position monitors } \\
\text { (BPMs) at each quadrupole }\end{array}$ \\
\hline Beam Profile Monitor & 2 planes & $\begin{array}{l}\text { Residual gas ionization profile monitor, } \\
\text { Two planes }\end{array}$ \\
\hline Beam Loss Monitors & 80 & $\begin{array}{l}\text { Glass ion chambers located at each } \\
\text { quadrupole and at significant loss points. }\end{array}$ \\
\hline Fast Beam Loss Monitors & 8 & $\begin{array}{l}\text { Photomultipliers used to look for losses } \\
\text { over a single turn or at extraction }\end{array}$ \\
\hline Beam In Gap Monitor & 1 & $\begin{array}{l}\text { A monitor of the beam which is outside the } \\
\text { RF bucket }\end{array}$ \\
\hline Tune Measurement System & 2 planes & Kicker excited, FFT analysis \\
\hline Beam Current Monitor & 1 & $\begin{array}{l}\text { Magnetic feedback type beam current } \\
\text { transformer }\end{array}$ \\
\hline Fast Current Monitor & 2 & $\begin{array}{l}\text { Fast beam current transformer to look at } \\
\text { bunch longitudinal density distribution }\end{array}$ \\
\hline
\end{tabular}


1. S. Schlöjl, K. Wittenburg, "A Beam Loss Monitor System for HERA", Proc Xvth Intl Conf on High Energy Accel., Hamburg 1992, p32

2. J. Balsamo et al., IEEE Trans. Nucl Sci., NS-24, $\underline{3}, 1807$ (1977)

3. E. R. Beadle, G. W. Bennett, and R. L. Witkover, "The AGS Booster Beam Loss Monitor System", Proc. 1991 Particle Accel. Conf. 91CH3038-7, p1231, 1991

4. R. L. Witkover, "A Microprocessor Based Beam Loss Monitor System", IEEE Trans. Nucl. Sci., NS-26, 3 , 3313 (1979)

5. R. E. Shafer, et al., "The Tevatron Beam Position and Beam Loss Monitoring Systems", Proc. 12th Intl. Conf on H. E. Accel., p609 (1983)

6. J. T. Hyman, "Toroidal Transformers and Pre-amplifiers for Transparent Beam Current Monitors",Report NIRL/R/30, Rutherford H. E. L. (1963)

7. K. Unser, "Beam Current Transformer with DC to $200 \mathrm{MHz}$ Range", IEEE Trans Nucl. Sci., NS-16, $\underline{3}, 934$ (1969)

8. M. Plum, Private communication, $1 / 8 / 97$

9. R. C. Webber, "Measurement Techniques Including Design of a Wall Current Monitor" Proceedings of the Beam Instrumentation Workshop 1989, p 85, AIP Conf. Proc. 212, AIP Press, NY

10.M. Plum, "Beam Diagnostics at High Intensity Storage Rings", Proceedings of the Beam Instrumentation Workshop 1993, p 72, AIP Conf. Proc. 319, AIP Press, NY

11. A. N. Stillman, T. Thern and R. L. Witkover, Rev. Sci. Instrum. 633412 (1992)

12. Howard L. Weisberg, AGS Division Technical Note No. 173, "Beam Profile Monitors for the AGS", July 8, 1981

13. Yan Yin, "Development on Multistrip Monitor for Nonintercepting Measurement of Beam Geometric Moments", Proc. 1993 Particle Accel. Conf. 93CH3279-7, p2441, 1993 